\title{
Burden of Chikungunya and its seasonal trend in south Karnataka- A study in a tertiary care centre
}

\author{
Trupti B. Naik ${ }^{1}$, Sathish J. V.,"*, Jayashree S. ${ }^{3}$ \\ ${ }^{\mathbf{1}}$ Assistant Professor, ${ }^{\mathbf{2}}$ Associate Professor, ${ }^{\mathbf{3}}$ Tutor, Dept. of Microbiology, Chamarajanagar Institute of Medical Sciences, \\ Chamarajanagar, Karnataka, India \\ *Corresponding Author: Sathish J. V \\ Email: javagalsathish37@gmail.com
}

Received: $2^{\text {nd }}$ August, 2018

Accepted: $17^{\text {th }}$ September, 2018

\begin{abstract}
Introduction: Mosquito borne arboviral infections have become major public health problem at present due to many emerging and re-emerging infections and Chikungunya is one of them. Although Chikungunya causes severe clinical manifestations like arthritis, it is not routinely tested at the health care facilities and therefore goes undiagnosed and as such its prevalence is likely to be underestimated. Climate is one of the important factor influencing the occurrence and distribution of disease.

Objectives: a) To estimate seroprevalence of Chikungunya virus infection among clinically suspected cases. b) To determine the seasonal variation of Chikungunya infection.

Materials and Methods: A cross sectional study was conducted at a tertiary care hospital using secondary data maintained in the microbiology laboratory registers, for clinically suspected patients of Chikungunya infection who reported to the hospital from January- December 2017. Prevalence was estimated by analyzing data for the results of IgM antibody by ELISA tests and any variations in disease reporting by age, gender and season were assessed. Statistical analysis was done using WHO Epi info software version 3.5.4.

Results: A total of 1308 serum samples were analyzed during the study period. Out of which $123(9.4 \%)$ samples were found positive for Chikungunya infection by IgM ELISA. The proportion of Chikungunya cases was higher in monsoon season with maximum rate of positivity in the month of July 48 (39.02\%). Age group of 16-30 years was the most commonly affected i.e. $(35.77 \%)$.

Conclusion: The present study confirms that Chikungunya is mainly a disease of rainy season and also identifies certain vulnerable groups for effective planning of interventions.
\end{abstract}

Keywords: Chikungunya, Immunoglobulin M, Seasonal variation, Seroprevalence.

\section{Introduction}

Arthropod-borne viral infections cause major disease burden in tropical and subtropical countries worldwide $^{1}$ and Chikungunya is one among them. Chikungunya virus (Arbovirus), a member of the genus Alpha virus and family Togaviridae is mainly transmitted by the bite of mosquito Aedes aegypti and less frequently by Aedes albopictus. ${ }^{2}$ Climate is an important factor contributing to change in the disease epidemiology. ${ }^{3}$ Studies recently reported in Southeast Asia, found that Chikungunya outbreaks were negatively correlated $(\mathrm{P}<0.05)$ with drought conditions, but positively correlated with warmer-thannormal temperatures and rainfall. ${ }^{4}$

India is severely affected with over 1.4 million infections as per recent estimates and the disease is known to cause epidemics when the proportion of immune naive population increases. By the latest report in 2010, Chikungunya has spread to more than 18 states/union territories within the country and the outbreak is still continuing. This rapid spread of Chikungunya demonstrated the devastating magnitude of infection and its ability of transmission in temperate regions. ${ }^{5}$ Resurgence has been attributed to various factors including urbanization, globalization, increase in the mosquito population, loss of herd immunity and the mutation $\mathrm{A} 226 \mathrm{~V}$ in the $\mathrm{E} 1$ gene causing a significant increase in CHIKV infectivity for Aedes albopictus. ${ }^{6}$

Chikungunya causes fever and nonspecific clinical manifestations like arthralgia, chills, headache, nausea, vomiting, low back pain, and rash lasting for a period of 1-7 days ${ }^{7}$ similar to malaria, dengue and other bacterial infections, but it is not routinely tested at the health care facilities and therefore goes undiagnosed and as such its prevalence is likely to be underestimated. ${ }^{8}$ The probable diagnosis of Chikungunya can be made on the basis of clinical manifestations, where as confirmatory diagnosis can be made only by laboratory tests. Currently, used tests include RT-PCR for confirming the presence of CHIKV, while sensitive IgM antibody ELISA is used to distinguish from dengue. ${ }^{9}$ Hence the present study was undertaken with the following objectives 1) To estimate seroprevalence of Chikungunya infection among clinically suspected cases 2) To determine the seasonal variation of Chikungunya infection.

\section{Materials and Methods}

The present cross-sectional study was conducted at a tertiary care hospital of Chamarajanagar Institute of Medical Sciences, Chamarajanagar using secondary 
data maintained in the microbiology laboratory registers, of clinically suspected chikungunya cases reported to various inpatient and out-patient departments of the hospital for a period of one year from January-December 2017, after obtaining prior permission from the head of the Institute. Prevalence was estimated by analyzing data for the results of IgM antibody by ELISA tests and any variations in disease reporting by gender, age and season were assessed.

Approximately $5 \mathrm{ml}$ of blood samples were collected from all the suspects of Chikungunya as a part of the routine laboratory work and the sera were separated by centrifugation of the whole blood sample and tested for the Chikungunya IgM antibody by using IgM antibody capture ELISA kit developed by National Institute of Virology (Arbovirus Diagnostic, NIV, Pune, India). Manufacturer's instructions were followed strictly while performing the ELISA. Values were calculated and results were interpreted as per manufacturer's guidelines.

Principle of IgM Capture ELISA for CHIK: Antihuman IgM are coated on to the solid surface (wells) and if IgM antibodies are present in the patient's sera, they are captured by it. If IgM and CHIK antigen, which is added in the next step, are homologous, the antigen binds to captured IgM. During the washing step unbound antigen is removed. Biotinylated anti-CHIK monoclonal antibody (CHIK-B) is added in the subsequent steps followed by avidin-histidine rich protein (HRP). Then substrate is added and development of color is monitored. $1 \mathrm{~N} \mathrm{H} 2 \mathrm{SO} 4$ is used to stop the reaction. The optical density (OD) is monitored at $450 \mathrm{~nm}$. OD values are directly proportional to the amount of CHIK virus specific IgM antibodies present in the sample. Kit controls and inhouse positive and negative controls were used to validate the test.

Statistical Analysis: Data was analyzed using WHO Epi info software version 3.5.4.

\section{Results}

A total of 1308 serum samples from suspected cases of Chikungunya were tested for presence of specific IgM antibodies by ELISA method and of which $123(9.4 \%)$ samples were found positive for Chikungunya. Majority i.e. $44(35.77 \%)$ of the positive cases were in the age group of 16-30 years and the proportion of positive cases was slightly higher in males with male to female ratio of 1:0.75. A seasonal peak of Chikungunya infection was seen in the months of July 48 (39.02\%) followed by June i.e. 31 (25.2\%).

Fig. 1: Illustrates seroprevalence of Chikungunya infection. Among 1308 clinically suspected cases, 123 (9.4\%) were found to be positive for Chikungunya infection by IgM ELISA in the present study.

Table 1 and Fig. 2: Of the 123 total positive cases of Chikungunya, though the number of positive cases 44 (35.77) was higher in the age group of 16-30 years, the rate of positivity was higher in the age group of 31-60 years $(23.02 \%)$. The study found no significant association between the prevalence of Chikungunya and the age group of patients $(\mathrm{P}>0.05)$.

Table 2: Gender wise distribution of samples of Chikungunya suspects and positives. Out of 1308 samples tested, $661(50.53 \%)$ were females and the rest males. Out of the 123 total positive cases of Chikungunya, both the proportion of cases i.e. 70 $(56.91 \%)$ and the rate of seropositivity i.e. $10.81 \%$ was slightly higher in males as compared to females. However, there was no significant association between the prevalence of Chikungunya and gender of patients $(\mathrm{P}>0.05)$.

Fig. 3 and Table 3: Seasonal trend showed that suspected cases of Chikungunya infection were highest in the month of June and July followed by August and slowly tapered by September with maximum positive cases in the month of July 48 (39.02\%) followed by June i.e. 31 (25.2\%). Serologically confirmed Chikungunya cases were highest i.e. 108 (87.8\%) during the monsoon period compared to other seasons of the year and it was found to be statistically significant $(\mathrm{P}<0.05)$.

Table 1: Age wise distribution of samples of Chikungunya suspects and positives

\begin{tabular}{|l|c|c|c|}
\hline \multirow{2}{*}{ Age in years } & Suspects & Positives & Rate of positivity \\
\cline { 2 - 4 } & No. $(\%)$ & No. $(\%)$ & \% \\
\hline$<15$ & $284(21.71)$ & $25(20.32)$ & 8.8 \\
\hline $16-30$ & $531(40.59)$ & $44(35.77)$ & 8.28 \\
\hline $31-45$ & $304(23.24)$ & $35(28.45)$ & 11.51 \\
\hline $46-60$ & $139(10.62)$ & $16(13)$ & 11.51 \\
\hline$>60$ & $50(3.82)$ & $3(2.43)$ & 6 \\
\hline Total & $1308(100)$ & $123(100)$ & 9.4 \\
\hline $\boldsymbol{x}^{2}=\mathbf{3 . 8 9} \mathbf{P}=\mathbf{0 . 4 2}$ &
\end{tabular}


Table 2: Gender wise distribution of Chikungunya suspects and positives

\begin{tabular}{|l|c|c|c|}
\hline Gender & Suspects & Positive & Rate of positivity \\
\hline & No. $(\%)$ & No. $(\%)$ & $(\%)$ \\
\hline Male & $647(49.46)$ & $70(56.91)$ & 10.81 \\
\hline Female & $661(50.53)$ & $53(43.08)$ & 8.01 \\
\hline Total & $1308(100)$ & $123(100)$ & 9.4 \\
\hline $\boldsymbol{x}^{2}=\mathbf{3 . 0 1} \mathbf{P}=\mathbf{0 . 0 8 2}$ & \multicolumn{4}{|l}{} \\
\hline
\end{tabular}

Table 3: Season wise distribution of Chikungunya suspects and positives.

\begin{tabular}{|l|c|c|c|}
\hline Months & Suspects & Positives & Rate of positivity \\
\hline & No. $(\%)$ & No. $(\%)$ & \% \\
\hline Pre Monsoon & $90(6.88)$ & $4(3.25)$ & 4.44 \\
\hline Monsoon & $1173(89.67)$ & $108(87.8)$ & 9.2 \\
\hline Post Monsoon & $45(3.44)$ & $11(8.94)$ & 23.91 \\
\hline Total & $1308(100)$ & $123(100)$ & 9.4 \\
\hline $\boldsymbol{x}^{\mathbf{2}=14.60} \mathbf{P}=\mathbf{0 . 0 0 0 6}$ \\
\hline
\end{tabular}

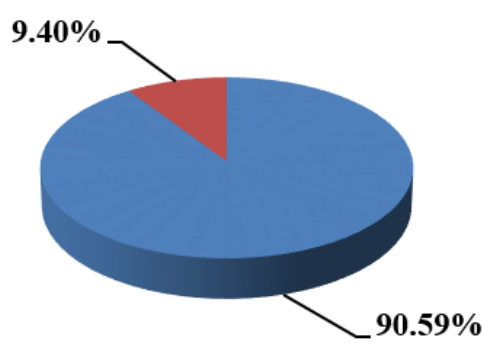

- Seronegativity

- Seropositivity

Fig. 1: Seroprevalence of Chikungunya infection

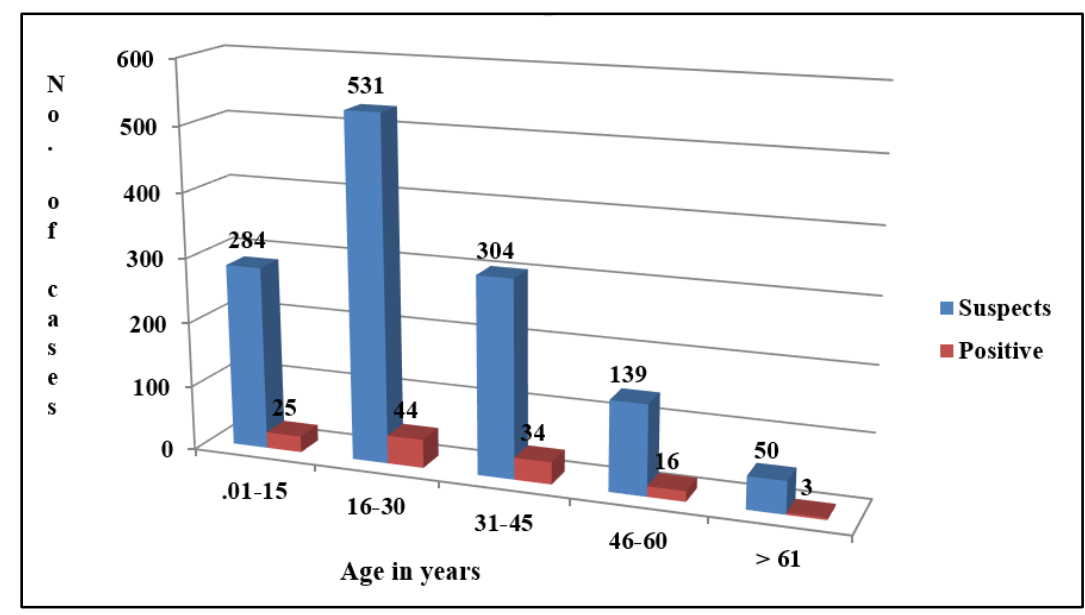

Fig. 2: Age wise distribution of Chikungunya suspects and positives 


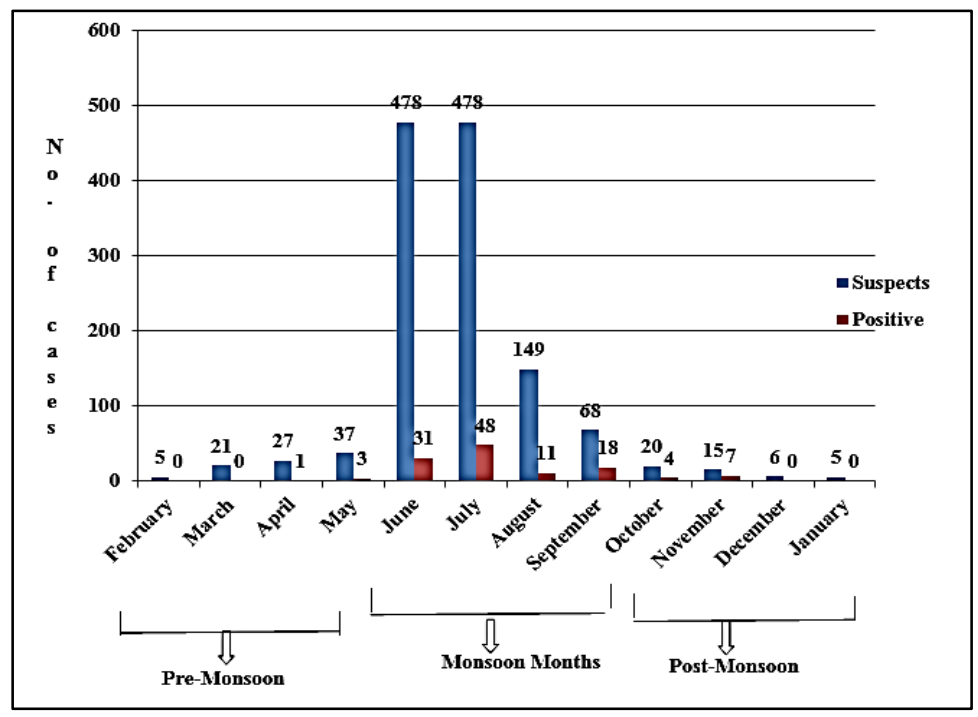

Fig. 3: Seasonal/ month wise distribution of Chikungunya suspects and positives

\section{Discussion}

As per literature, chikungunya virus is reported from nearly 40 countries, and listed as a category C priority pathogen of US National Institute of Allergy and Infectious Diseases (NIAID) in 2008..$^{10}$ Earlier it was not considered as a life threatening infection but recent epidemiological evidence has shown the case fatality rate of around $0.1 \% .^{11}$ After a quiescence of about three decades, outbreak of Chikungunya is being reported from different parts of India. The present study confirms and extends the findings of recent reports from India and other parts of world indicating a reemergence of severe chikungunya disease which is becoming a major public threat. Prevalence of chikungunya in the present study is $9.4 \%$ which is well correlated with other studies. ${ }^{1,5,12,13}$ while some other studies have reported higher seroprevalence. ${ }^{14-22}$

In our study maximum positive cases of chikungunya were seen in the age group of 16-30 years i.e. $(35.77 \%)$ followed by $31-45$ years i.e. $(28.45 \%)$ similar to the findings of other studies. ${ }^{1,5,12,18}$ This may be explained with the fact that this age group of people either go for education or work at day time and the main vector (Aedes aegypti) has day biting habit. On the contrary some studies have reported $31-45$ years as the most affected age group. ${ }^{14,15}$

The present study showed male $70 \quad(56.91 \%)$ preponderance over female $53(43.08 \%)$ with sex ratio of male to female 1:0.75, which could be attributed to the social bias in health seeking behavior generally observed against females rather than to disease susceptibility based on gender. This finding is correlating with studies done by Kalawat $\mathrm{U}$ et al. ${ }^{23}$ Various other studies conducted worldwide have shown inconsistencies on gender bias to disease susceptibility with few studies reporting both genders to be equally susceptible. ${ }^{17,24-26}$
Seasonal trend of chikungunya infection was analyzed by the month wise data of samples. In India the first CHIKV outbreak in 1963 was observed during July to December, coinciding with the monsoon and post monsoon seasons. However, in the present study, CHIKV was detected more during the monsoon season with positive cases being raised gradually from June with peak in the month of July and declined in the month of October. This finding correlated with findings of Tomar A. et al. at Navi Mumbai, ${ }^{1}$ Bharti $\mathrm{N}$ et al. at Tamil Nadu ${ }^{27}$ Nandi J. et al. at Delhi, ${ }^{28}$ Ray P. et al. in a multicentric study ${ }^{19}$ Divya P. et al. at Ballari ${ }^{18}$ Manimunda SP et al. at Dakshin Kannada ${ }^{29}$ and Mohanty I et al. at Southern Odissa. ${ }^{7}$ This may be due to increased transmission of disease at the start of the rainy season with increased breeding of both Aedes aegypti and Aedes albopictus. Higher humidity lengthens their life span and increased temperatures shortens the extrinsic incubation period. As mosquitoes are sensitive to changes in temperature and available moisture, they decrease in number in dry and cool seasons so cases declined in rest of the year. Certain studies showed highest number of cases during July to December (Ahmedabad) ${ }^{22}$ September-December $\left(\right.$ Lucknow) ${ }^{12}$ November ${\text { (Varanasi })^{5}}^{5}$ sept- nove (Mumbai) $^{14} \quad$ sep-dec $\quad(\text { Ahmedabad })^{15}$ sep-jan (Ahmedabad). ${ }^{16}$ These seasonal peaks emphasize the need to create public awareness and undertake necessary vector control measures including improvements in sanitation and hygiene during pre monsoon period.

\section{Conclusion}

The seroprevalence of Chikungunya in our study was $9.4 \%$ which reiterates the fact that Chikungunya is a major health concern in our setting. The present study also confirms that Chikungunya is mainly a disease of 
monsoon and identifies certain vulnerable groups for effective planning of interventions.

\section{References}

1. Tomar A, Hodiwala AB, Khiste DD. Prevalence of Chikungunya viral infection in a tertiary care hospital, Navi Mumbai, Maharashtra. JMSCR. 2017;05(01):1594815951.

2. Panning M, Hess M, Fischer W, Grywna K, Pfeffer M, Drosten C. Performance of the Real Star Chikungunya virus Real-Time Reverse Transcription-PCR Kit. J. Clin. Microbiol. 2009;47(9):3014-16.

3. Weaver SC, Reisen WK. Present and future arboviral threats. Antiviral Res. 2010;85(2):328-45.

4. Anyamba A, Linthicum KJ, Small JL, Collins KM, Tucker CJ, Pak EW, Britch SC, Eastman JR, Pinzon JE, Russell KL. Climate teleconnections and recent patterns of human and animal disease outbreaks. PLoS Negl Trop Dis. 2012;1:1465.

5. Dinkara A, Singh J, Prakasha P, Dasa A, Nath G. Hidden burden of chikungunya in North India; A prospective study in a tertiary care centre. J Infect Public Health. 2017;813-819.

6. Kumar NP, Suresh A, Vanamail P, Sabesan S, Krishnamoorthy KG, Mathew J, Jose VT, Jambulingam P. Chikungunya virus outbreak in Kerala, India, 2007: a seroprevalence study. Mem Inst Oswaldo Cruz. 2011;106(8):912-916.

7. Mohanty I, Dash M, Sahu S, Narasimham MV, Panda P and Padhi S. Seroprevalence of Chikungunya in Southern Odisha. J Family Med Prim Care. 2013;2(1):33-36.

8. Mwongula AW, Mwamburi LA, Matilu M, Siambaand DN, Wanyama FW. Seroprevalence of Chikungunya infection in pyretic children seeking treatment in Alupe District Hospital, Busia county Kenya. IJCMAS. 2013;2(5):130-139.

9. Galate LB, Agrawal SR, Shastri JS, Londhey V. Chikungunya fever among patients with acute febrile illness attending a tertiary care hospital in Mumbai. $J$ Lab Physicians. 2016;8(2):85-9.

10. Gandhi BS, Kulkarni K, Godbole M, Dole SS, Kapur S, Satpathy P, Khatri AM, Deshpande PS, Azad F, Gupte N, Bharadwaj R, Bollinger RC, Gupta A. Dengue and chikungunya co-infection associated with more severe clinical disease than mono-infection. Int $J$ Healthc Biomed Res. 2015;3(3):117-23.

11. Furuya-Kanamori L, Liang S, Milinovich G, Soares Magalhaes RJ, Clements AC, Hu W, Brasil P, Frentiu FD, Dunning R, Yakob L. Distribution and co infection of chikungunya and dengue viruses. BMC Infect Dis. 2016;16(March):84.

12. Shruti M, Krunal M, Singh KP, Dhole TN. Emergence of Chikungunya infection in North India. APALM. 2016;3:314-319.

13. Gandhi BS, Kulkarni K, Godbole M, Dole SS, Kapur S, Satpathy P, Khatri AM, Deshpande PS, Azad F, Gupte N. Bharadwaj R, Bollinger RC, Gupta A. Dengue and Chikungunya co-infection associated with more severe clinical disease than mono-infection. Int J Healthc Biomed Res. 2015;3(3):117-123.

14. Baveja S, Habeeb SH, Ramchandran A, Turbadkar D, Kumar CA, Randive M. Seroprevalence of Chikungunya cases in a tertiary care hospital in Mumbai. Med Int $J$ of Microbiology. 2017;4(1):01-03.

15. Sodhatar K, Vegad MM, Patel D, Kinariwala D, Patel P, Sarita. Seroprevalence and epidemiology of Chikungunya with it's seasonal trends diagnosed at tertiary care hospital, Ahmedabad. NJIRM. 2017;8(2):20-22.

16. Sakhiya AJ, Gamit M, Prajapati K, Patel D, Shah P. Seroprevalence of chikungunya cases in a tertiary-care hospital in Ahmedabad. Int J Med Sci Public Health. 2015;4:1297-1300.

17. Kawle AP, Nayak AR, Bhullar SS, Borkar SR, Patankar SD, Daginawala HF, Singh LR, Kashyap RS. Seroprevalence and clinical manifestations of chikungunya virus infection in rural areas of Chandrapur, Maharashtra, India. J Vector Borne Dis 2017;54:35-43.

18. Divya P, Krishna S. Seroprevalence of Chikungunya virus infection in Ballari and nearby districts of Karnataka IJMMTD. 2016;2(4):175-177.

19. Ray P, Ratagiri VH, Kabra SH, Lodha R, Sharma S, Sharma BS, Kalaivani M, Wig N. Chikungunya infection in India: results of a prospective hospital based multicentric study. PLOS ONE. 2012;7(2):1-8.

20. Barve S., Nanda S, Javadekar TB. Chikungunya fever: the resurgence and epidemiological pattern in western India. NJMR 2013;3(2):159-161.

21. Chattopadhyay S., Mukherjee R., Nandi A. and Bhattacharya N. Chickungunya virus infection in West Bengal, India. Indian J Med Microbiol. 2016;34(2):213215.

22. Modi KP, Patel DA, Vegad MM, Mistry AU, Padaria NJ, Rathod AB. Sero-prevalence of Dengue and Chikungunya, their co-infection and seasonal trends of these infections at a tertiary care hospital, Ahmedabad, Gujarat. IJMR. 2017;9(1):819-822.

23. Kalawat U, Sharma K, Reddy GS. Prevalence of Dengue and Chikungunya fever and their Co- infection. Indian $J$ Pathol Microbiol. 2011;54(4):844-846.

24. Renault P, Solet JL, Sissoko D, Balleydier E, Larrieu S, Filleul L, Lassalle C, Thiria J, Rachou E, Valk H, Ilef D, Ledrans M, Quatresous I, Quenel P, Pierre V. A major epidemic of chikungunya virus infection on Reunion Island, France, 2005-2006. Am J Trop Med Hyg. 2007;77: 727-31.

25. Sebastian MR, Lodha R, Kabra SK. Chikungunya infection in children. Indian J Pediatr. 2009;76:185-9.

26. Sergon K, Yahaya AA, Brown J, Bedja SA, Mlindasse M, Agata N, Allaranger Y, Ball MD, Powers M, Ofula V, Onyango C, Konongoi LS, Sang R, Njenga NK, Breiman RF. Seroprevalence of Chikungunya virus infection on Grande Comore Island, union of the Comoros, 2005. Am J Trop Med Hyg. 2007;76:1189-93.

27. Bharti N, Ramakritinan C. M, Kolandaswamy K. Geographical information system based study on Dengue and Chikungunya at Western Ghats districts, Tamil Nadu, India. Int J Mosq Res. 2015;2(3):30-38.

28. Nandi J, Sharma RS, Datta PK, Dhillon GPS. Dengue in the National Capital Territory (NCT) of Delhi (India): epidemiological and entomological profile for the period 2003-2008. Dengue Bull. 2008;32:156-61.

29. Manimunda SP, Sugunan AP, Rai SK, Vijayachari P, Shriram AN, Sharma S, Muruganandam N, Chaitanya IK, Guruprasad DR, Sudeep AB. Short Report : Outbreak of Chikungunya Fever, Dakshina Kannada District, South India. 2008. Am J Trop Med Hyg. 2008;83(4):751-754.

How to cite this article: Naik T. B, Sathish J. V, Jayashree S. Burden of Chikungunya and its seasonal trend in south Karnataka- A study in a tertiary care centre. Indian J Microbiol Res. 2018;5(4):492-496. 